\title{
Identification of AFLP Markers Favorable to Heterosis in Hybrid Rice
}

\author{
Xiaochuan Liu ${ }^{* 1)}$ Koshun Ishiki ${ }^{2}$ and Weixia Wang1) \\ 1) China National Rice Research Institute, Hangzhou 310006, Zhejiang, P. R. China \\ 2) Japan International Research Center for Agricultural Sciences, Okinawa Subtropical Station, Maezato, Ishigaki, Okinawa 907-0002, \\ Japan
}

In hybrid rice breeding programs, it is the most important to select donors for improving parental lines and to predict the hybrid performance. In this study, AFLP marker technology and a half-diallel method were used to address these related issues in hybrid rice. Based on the representative heterosis characters representing in the $F_{1}$ generation, an innovative method was designed to identify the genetic diversity related to heterosis for yielding with the detected AFLP. The results showed that the genetic diversity among the parental lines was certainly related to $F_{1}$ heterosis, and a few loci contributed to hybrid vigor. We discovered 11 favorable alleles with different genetic effects significantly contributing to the heterosis in grain yield among 85 AFLP markers, and 8 unfavorable alleles that significantly reduced heterosis. Based on the favorable and unfavorable alleles found, 42 accessions were evaluated for the exploitation potentials of heterosis, as reference for hybrid rice breeding. Moreover, some aspects related to heterosis formation are discussed in this paper.

Key Words: AFLP markers, heterosis, rice.

\section{Introduction}

In heterosis, $F_{1}$ plants usually have higher vigor than their parental lines. Over the decades, it has been widely used in a number of crops. It has greatly contributed to agricultural production worldwide by increasing the yield and broadening adaptability of hybrid varieties. Heterosis has been of interest to plant breeders for a long time, especially in both selecting the donors for parental lines and predicting the hybrid performance.

Out of the large number of combinations annually made and evaluated in hybrid breeding programs, only a few hybrids are selected. This means that extremely intensive labor is required, time is consumed and the selection efficiency is low. Thus, it is important to explore alternative approaches to enhance the selection efficiency and to predict hybrid performance, such as through combining ability anal-

Communicated by T. Sasaki

Received November 22, 2001. Accepted April 2, 2002.

*Corresponding author (e-mail: xcliu@mail.hz.zj.cn) ysis and genetic distance on the basis of the agronomic traits of the parental lines. The results showed that these methods were useful for hybrid breeding programs (Dudley 1984, Zanoni et al. 1989, Virmani 1994). However, they are still impracticable due to the requirement of many crosses and extensive field tests. Isozyme analysis also revealed some isozyme loci associated with heterosis, but it was not informative enough to be used (Peng et al. 1988, Peng et al. 1991)

A number of genes conferring agronomic traits have been tagged with DNA markers (Wu and Tanksley 1993, Nagato et al. 1998). DNA markers have also been used for tagging the heterotic trait in various crops. Zhang et al. (1996) reported the importance of the genetic diversity of parental lines including heterozygosity and homozygosity for heterosis formation. The means of homozygous marker genotypes for yield at the inbred level significantly correlated with the hybrid level, but this correlation was not high enough to accurately predict hybrid performance (Dudley et al. 1992). The study using RFLP markers showed a strong linear correlation between heterozygosity and heterosis (Stuber 1992, Xiao et al. 1996). Some studies indicated that the DNA marker based genetic diversity was not of any predictive value for hybrid performance (Melchinger et al. 1990, Martin et al. 1995, Diers et al. 1996, Cerna et al. 1997). QTL analysis revealed that the major genetic basis of heterosis in rice was the dominance (Xiao et al. 1995). Some domains contributing to heterosis flanked by RFLP markers were found on several chromosomes (Xiao et al. 1996). The analysis of the interaction between different loci showed epistasis as an important factor controlling heterosis in rice (Yu et al. 1997). Those studies were carried out based on the $\mathrm{F}_{2}$ segregating and recombining principles for tagging homozygotic traits whereas heterotic traits mainly represented under $\mathrm{F}_{1}$ heterozygosity.

In this study, we considered that heterosis was caused by the combining ability at different marker loci between the male and female parents. For parental improvement and heterosis prediction with DNA markers, an identification of the molecular markers for the specific genes contributing to heterosis in heterozygosity may be more important than that in homozygosity or QTLs (Liu and Wu 1998). Also, it is difficult to find a relationship between the general heterozygosity and heterotic traits though heterozygosity at some specific loci significantly contributed to heterosis. For this reason, we examined the genetic diversity in a set of male sterile and 
restorer lines using amplified fragment length polymorphism (AFLP) markers, to identify favorable and unfavorable DNA markers through correlation analysis between the polymorphism of parents and the yield levels of their hybrids under diallel-crossing trial, and analyzed their genetic effects on heterosis.

\section{Materials and Methods}

\section{Materials and Half-diallel crossing trial}

A half-diallel experiment was designed to obtain field data of grain yield at the China National Rice Research Institute (CNRRI), Hangzhou, P. R. China. The male and female lines in indica rice were collected and purified by bagging in two rice-growing seasons. A total of 6 elite and 2 inferior male sterile lines were crossed with 7 elite and 3 inferior restorers. A full set of 80 hybrids were made. Their performance was evaluated as later-season rice with a common cultivated management. They had normal fertility because of indica/indica hybridization and also they had a similar growth duration of 118-121 days in the later season rice. All of the plots were harvested on the same day. The total grain yield of each plot (200 plants) was recorded for further analysis.

A total of 42 samples including varieties, landraces and parental lines representing major parental lines for hybrid rice in China were used for detecting genetic diversity and predicting their heterotic potentials.

\section{DNA Preparation}

Using the method described by Maroof et al. 1984, DNA samples were extracted from $5.0 \mathrm{~g}$ young leaves of the parental lines with twice extractions in chloroform/isopentyl alcohol $(24: 1)$. They were soaked in $70 \%$ ethanol for storage at $-20^{\circ} \mathrm{C}$. DNA samples were dissolved in TE buffer and determined for quality and quantity. Finally the samples were diluted in $100 \mathrm{ng} / \mu \mathrm{l}$ with $\mathrm{TE}_{0.1}$ buffer for further analysis.

\section{AFLP Assay}

A total of 22 combinations of AFLP primers with EcoRI+3/MseI+3 were used in this study. AFLP analysis was carried out following the protocol described in the product manual (AFLPTM Analysis System I, GiBCO BRL Life Technologies, USA) with some modification depending on different primers. Briefly, 300 ng genome DNA was digested with EcoRI and MseI, ligated to their supplied adapters and PCR amplified by the following two steps. In the preamplification, the primers of $E c o \mathrm{RI}+\mathrm{A} / M s e \mathrm{I}+\mathrm{C}$ were used. In the selective amplification, the samples were prepared by diluting 20-100 times from the pre-amplified samples. Then, the selectively amplified samples were loaded on $10 \%$ polyacrylamide gels with the $140 \mathrm{~mm}$ high, $140 \mathrm{~mm}$ wide and 1.0 mm thick size for separating DNA fragments under the condition of 300 constant voltages and 120 minutes. Finally, the gel was visualized by silver staining (Silver stain II Kit,
Wako), and was recorded by photography.

\section{Statistical Analysis}

AFLP bands were scored as present " 1 " and absent "2" at the same position. Genetic diversity analysis was made with STATISTICA (Release 4.0 Statsoft Inc., Tulsa, OK, USA). Dendrograms were made using a cluster procedure with unweighted pair-group average (UPGA) and percentage disagreement.

A statistical innovative method was designed to identify favorable AFLP markers to heterosis based on both AFLP data of the parental lines and field data of their $F_{1}$ hybrid performance. It was assumed that if a pair of female and male parents is not polymorphic at one allele, their hybrid is considered as a "homozygote" (a control) though it is a certain heterozygosity which may produce heterosis. On the contrary, if it presents AFLP at the same allele, their hybrid is taken into account as a heterozygote. As a comparison of the heterozygotes with the corresponding "homozygotes" in their correspondent hybrid yield, its correspondent AFLP marker is defined as a favorable marker if the heterozygotes have a significantly higher yield than the "homozygotes". The statistical analyses were carried out using a specific computer software made by X.C. Liu and P.Q.Y. Tang.

\section{Results}

Using 22 combinations of AFLP primers, a total of 85 alleles were scored from 42 parental genotypes. Based on the cluster analysis of those alleles, the genetic diversity among 42 parental genotypes clearly showed 3 major groups (Fig. 1). Groups "A" and "B" mainly consisted of the male lines whereas group " $C$ " involved almost all female lines. The other accessions were derived from indica/japonica hybridization, away from the groups.

Eight female and ten male parents were used for tagging heterotic traits. The genetic diversity of those parents demonstrated a distinct difference between the female and male groups with the exception of Xianjing No. 1 (Fig. 2). The analysis of 5612 heterozygotes as compared with 7988 homozygotes detected within 85 AFLP alleles in the parental lines and the performance of their correspondent hybrids clearly revealed that 11 favorable alleles significantly contributed to heterosis. Meanwhile, 8 unfavorable alleles were found to significantly reduce heterosis (Table 1 and Table 2). Further analysis of the favorable alleles showed that those favorable alleles had different genetic effects on heterosis involving the additive (Fig. 3A), the dominant (Fig. 3B and Fig. 3C) and the over-dominant (Fig. 3D).

Forty-two different accessions were examined for these favorable and unfavorable alleles, and their effect on heterosis was approximately evaluated (Table 3 ).

\section{Discussion}

There is no doubt that a heterotic trait exists only in het- 


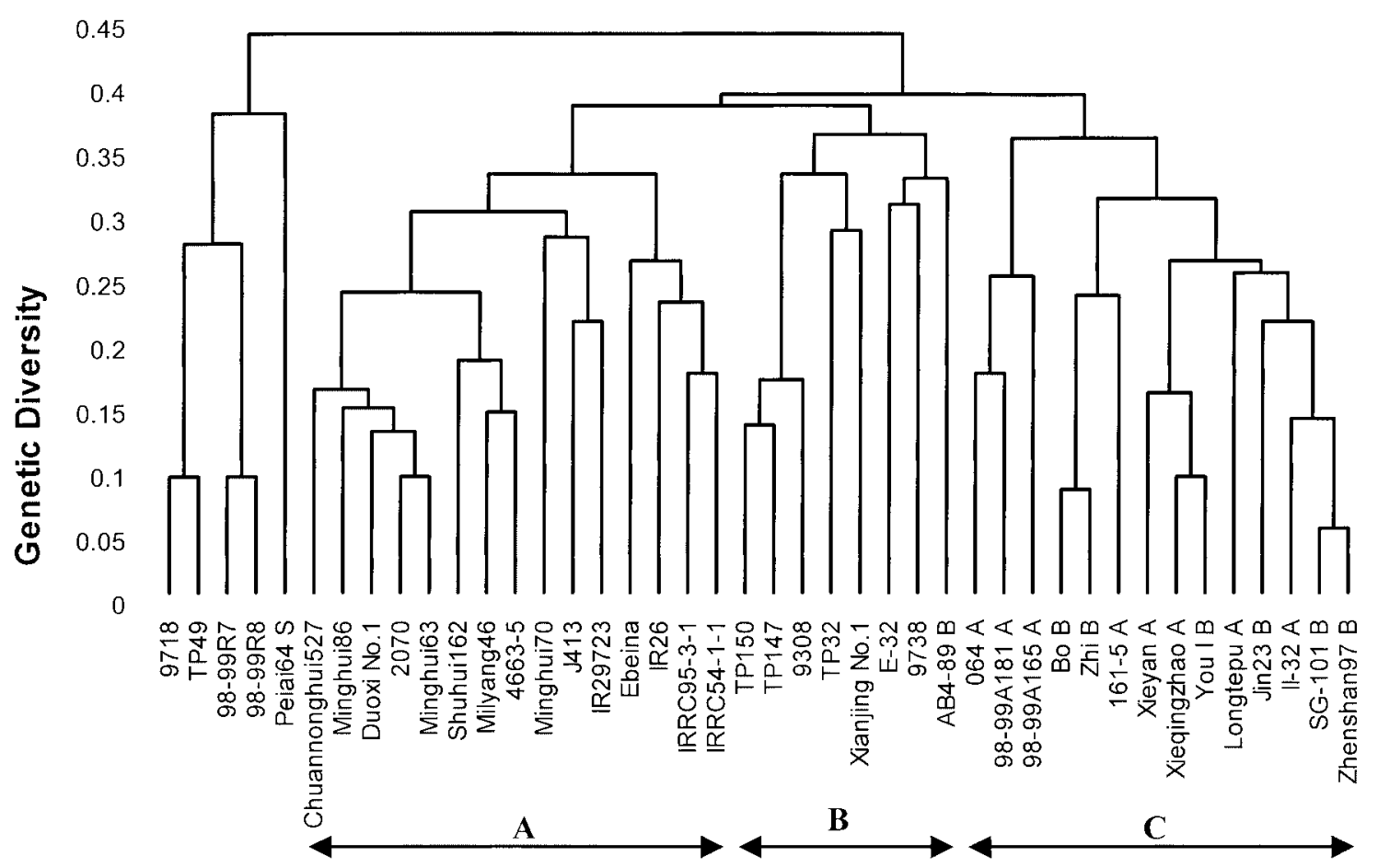

Fig. 1. Cluster diagram of the genetic diversity of 42 parental genotypes in rice constructed with 22 combinations of AFLP primers.

erozygosity, mainly in the $\mathrm{F}_{1}$ generation. In general, genetic diversity should be an important basis for heterotic formation though different results have been reported previously in various crops. So, it was still necessary to clarify the genetic diversity among the 42 accessions in this assay. From the results obtained using AFLP markers, 42 parental genotypes were clearly divided into 3 major groups (Fig. 1). Interestingly, the hybrids made from Group C and Group A are mainly used in rice production on a large scale at present, and the hybrids from Group C and Group B are very prom-

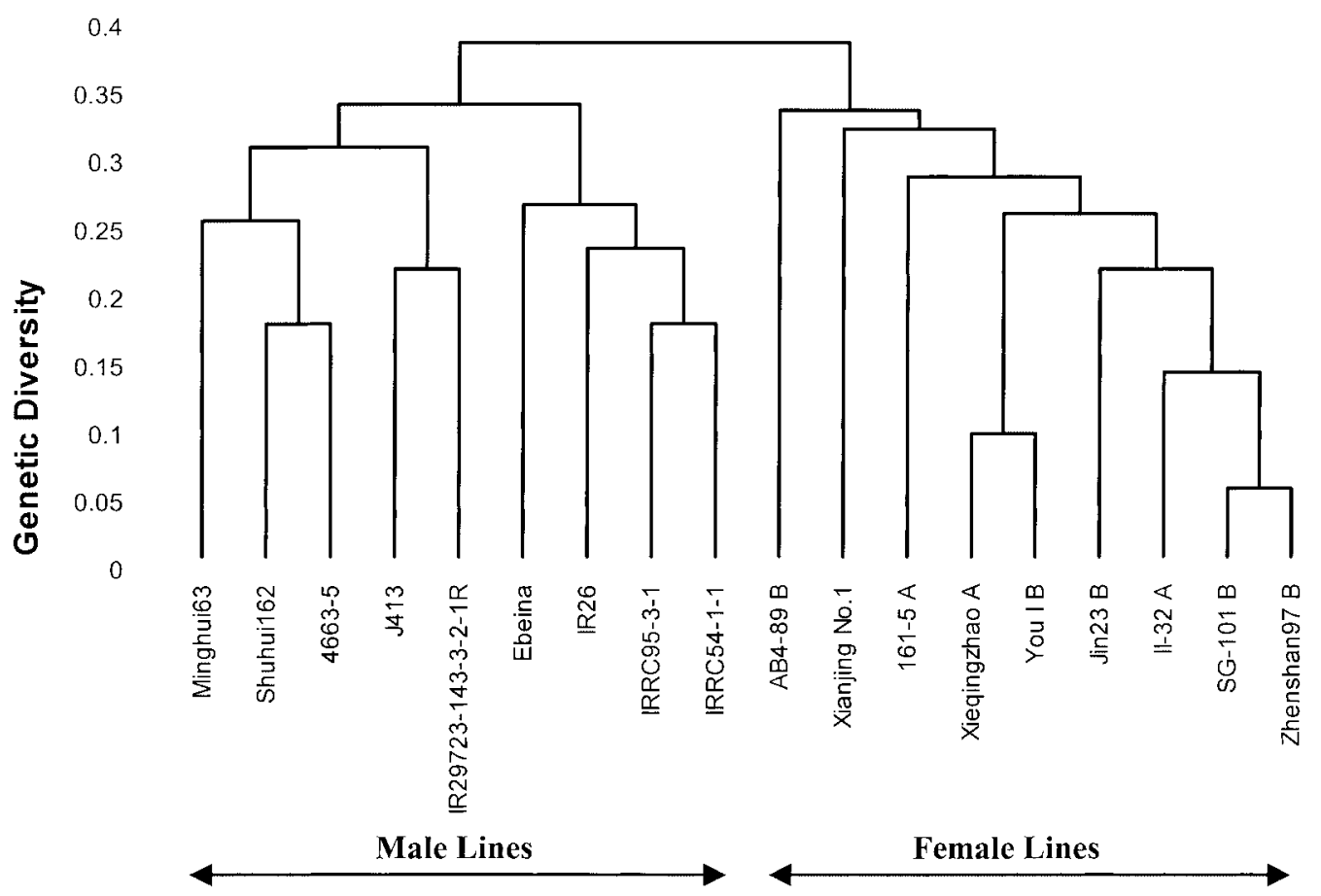

Fig. 2. Cluster diagram of the genetic diversity of the female and male parental lines used for tagging heterotic trait. 

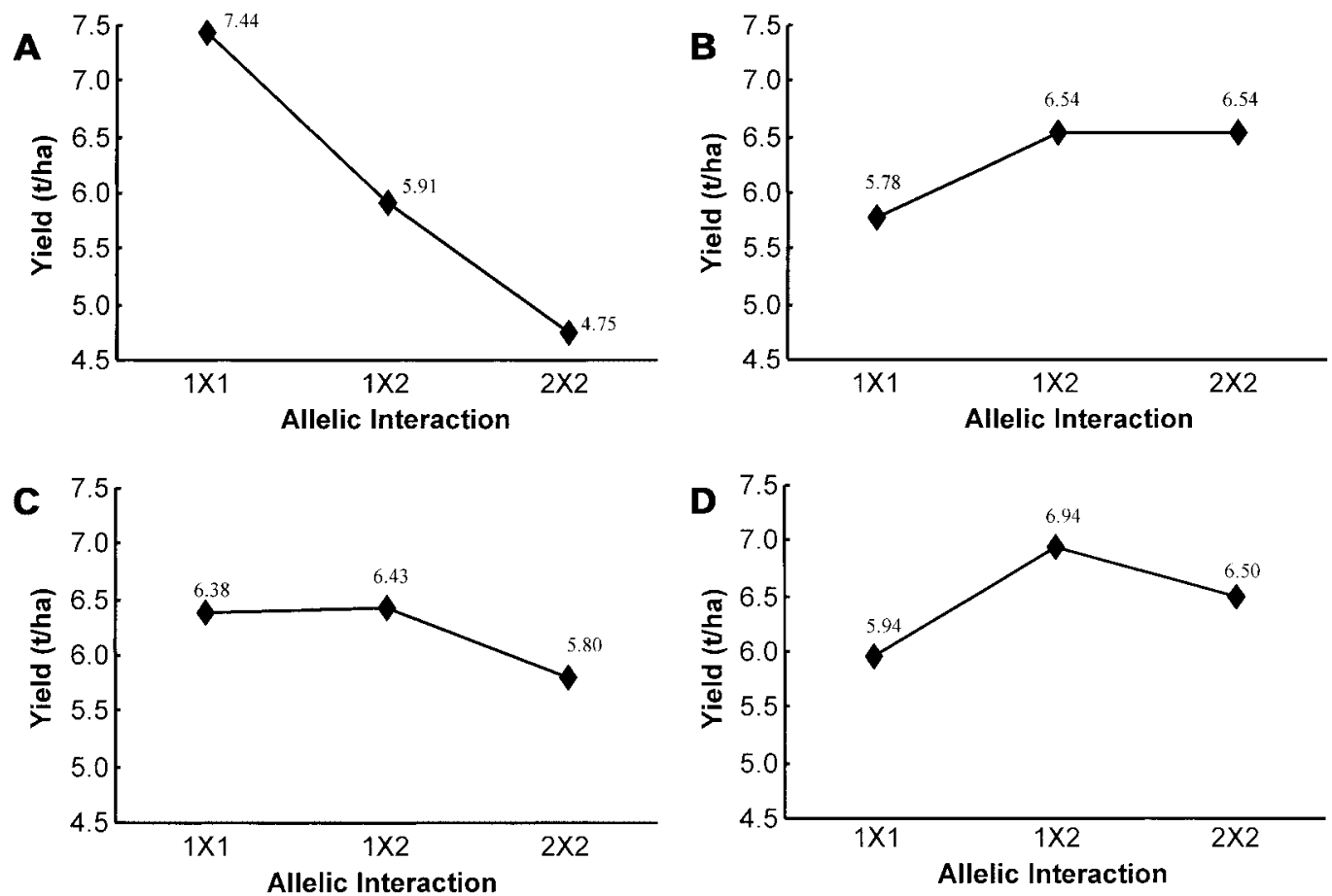

Fig. 3. Genetic effects resulting from the interactions of two alleles detected in AFLP analysis. A: additive effect detected by E-AAC/M-CAG primers. B: dominant effect by E-AAG/M-CAG primers.C: dominant effect by E-ACA/M-CTT primers. D: over-dominant effect by E-AGG/M-CAC primers.

ising for production. The other 5 accessions derived from indica/japonica hybridization have been developed for the super-hybrid rice. Further analysis of each group showed that the average discriminating index in group $\mathrm{A}$ is about 0.237, which is much lower than that in group $\mathrm{B}(0.307)$, but larger than that in group $\mathrm{C}(0.220)$. Based on these findings, we concluded that broadening the genetic diversity of parental lines could effectively increase heterosis.

However, broadening the genetic diversity is not always effective in increasing heterosis in practice because a number of unfavorable genes are also incorporated into the

Table 1. Favorable alleles detected by AFLP primers significantly contributing to heterosis in rice

\begin{tabular}{rcccc}
\hline \hline $\begin{array}{c}\text { Series } \\
\text { number }\end{array}$ & Primer combination $\begin{array}{c}\text { Molecular } \\
\text { weight } \\
\text { (Approx. bp) }\end{array}$ & T-value & P-value \\
\hline 50 & E-ACA/M-CAC & 1300 & 4.2365 & $0.0001^{* *}$ \\
90 & E-AAC/M-CAG & 450 & 3.4112 & $0.0008^{* *}$ \\
87 & E-AAC/M-CAG & 850 & 3.0978 & $0.0023^{* *}$ \\
79 & E-AGG/M-CAC & 170 & 2.9662 & $0.0035^{* *}$ \\
71 & E-ACC/M-CAA & 350 & 2.7336 & $0.0070^{* *}$ \\
83 & E-AGG/M-CTT & 450 & 2.6676 & $0.0084^{* *}$ \\
5 & E-ACG/M-CTT & 1500 & 2.5653 & $0.0112^{*}$ \\
92 & E-AAG/M-CAG & 1000 & 2.5387 & $0.0121^{*}$ \\
96 & E-ACA/M-CTT & 1150 & 2.3121 & $0.0221^{*}$ \\
76 & E-ACC/M-CTG & 170 & 2.1398 & $0.0339^{*}$ \\
9 & E-ACG/M-CTG & 1500 & 2.0917 & $0.0381^{*}$ \\
\hline
\end{tabular}

* Significant at $5 \%$ level.

** Significant at $1 \%$ level. elite lines. Moreover, there may be only a small difference in a genetic case related to heterosis between the male and female lines. It is the most important to understand what kind of genetic diversity should be broadened or avoided for exploiting super-heterosis. It should be extremely useful for parental selection and heterosis prediction to identify those parts of genetic diversity with molecular markers in hybrid breeding programs. An AFLP method has been approved to be useful for addressing this critical issue.

As a heterotic trait presenting under heterozygosity completely differs from any other agronomic trait under homozygosity, it might not be precisely tagged by means of $\mathrm{F}_{2}$ segregation and backcrossed populations though there were a number of valuable studies on heterosis using those

Table 2. Unfavorable alleles detected by AFLP primers significantly contributing to heterosis in rice

\begin{tabular}{ccccc}
\hline \hline $\begin{array}{c}\text { Series } \\
\text { number }\end{array}$ & Primer combination & $\begin{array}{c}\text { Molecular } \\
\text { weight } \\
\text { (Approx. bp) }\end{array}$ & T-value & P-value \\
\hline 42 & E-AAG/M-CAC & 380 & 3.9444 & $0.0001^{* *}$ \\
89 & E-AAC/M-CAG & 650 & 3.7639 & $0.0002^{* *}$ \\
14 & E-AGC/M-CAT & 100 & 3.1895 & $0.0017^{* *}$ \\
62 & E-ACT/M-CAT & 2000 & 2.733 & $0.0070^{* *}$ \\
36 & E-AAC/M-CAC & 250 & 2.7209 & $0.0072^{* *}$ \\
34 & E-AAC/M-CAC & 1100 & 2.6633 & $0.0085^{* *}$ \\
66 & E-AGC/M-CTT & 620 & 2.3019 & $0.0226^{*}$ \\
44 & E-ACA/M-CAA & 2000 & 2.0852 & $0.0387^{*}$ \\
\hline
\end{tabular}

* Significant at $5 \%$ level.

** Significant at $1 \%$ level. 
Table 3. The number and genetic effects of alleles of accessions favorable and unfavorable to heterosis among a total of alleles revealed by AFLP markers

\begin{tabular}{|c|c|c|c|c|}
\hline \multirow{2}{*}{ Accessions } & \multicolumn{2}{|c|}{ Favorable alleles } & \multicolumn{2}{|c|}{ Unfavorable alleles } \\
\hline & Number & Effects & Number & Effects \\
\hline Zhenshan97 B & 6 & 2.41 & 4 & 1.90 \\
\hline $\operatorname{Jin} 23 \mathrm{~B}$ & 6 & 2.77 & 4 & 2.02 \\
\hline You I B & 6 & 2.93 & 1 & 0.51 \\
\hline $161-5 \mathrm{~B}$ & 6 & 3.01 & 3 & 1.51 \\
\hline SG-101B & 5 & 2.44 & 3 & 1.51 \\
\hline AB4-89 B & 4 & 1.92 & 2 & 1.13 \\
\hline II-32 A & 6 & 2.80 & 3 & 1.38 \\
\hline Xieyan A & 7 & 3.31 & 3 & 1.28 \\
\hline Zhi B & 6 & 2.82 & 3 & 1.53 \\
\hline Bo B & 6 & 2.83 & 3 & 1.53 \\
\hline Peiai64 S & 5 & 2.41 & 4 & 1.94 \\
\hline Xieqingzhao A & 7 & 3.46 & 3 & 1.44 \\
\hline Longtepu A & 5 & 2.59 & 4 & 1.82 \\
\hline 98-99A165 A & 6 & 2.98 & 4 & 1.85 \\
\hline 98-99A181 A & 6 & 2.77 & 1 & 0.54 \\
\hline $064 \mathrm{~A}$ & 8 & 3.96 & 0 & 0.00 \\
\hline IRRC-54-1-1 & 6 & 3.19 & 4 & 1.73 \\
\hline IRRC-95-3-1 & 6 & 2.89 & 4 & 1.73 \\
\hline IR29723 & 6 & 2.90 & 0 & 0.00 \\
\hline IR26 & 6 & 2.91 & 4 & 1.73 \\
\hline Ebeina & 6 & 2.98 & 2 & 0.78 \\
\hline $\mathrm{J} 413$ & 5 & 2.42 & 0 & 0.00 \\
\hline Xianjing No. 1 & 4 & 1.99 & 3 & 1.51 \\
\hline $4663-5$ & 4 & 2.41 & 3 & 1.46 \\
\hline Minhui63 & 5 & 2.53 & 4 & 1.73 \\
\hline Minhui70 & 4 & 2.27 & 2 & 0.79 \\
\hline Shuhuil62 & 4 & 2.18 & 2 & 0.95 \\
\hline Duoxi No. 1 & 5 & 2.56 & 5 & 2.11 \\
\hline Chuanlonghui527 & 5 & 2.57 & 4 & 1.84 \\
\hline Milyang46 & 3 & 1.96 & 5 & 2.24 \\
\hline $98-99 \mathrm{R} 8$ & 5 & 2.64 & 5 & 2.45 \\
\hline 98-99R7 & 7 & 3.51 & 5 & 2.46 \\
\hline 9718 & 6 & 3.06 & 4 & 2.07 \\
\hline Minhui86 & 8 & 4.14 & 5 & 2.24 \\
\hline 9308 & 6 & 3.08 & 4 & 2.05 \\
\hline 2070 & 6 & 3.02 & 6 & 2.75 \\
\hline ТР147 & 7 & 3.55 & 3 & 1.67 \\
\hline TP150 & 6 & 2.84 & 3 & 1.67 \\
\hline ТР32 & 7 & 3.52 & 1 & 0.61 \\
\hline ТР49 & 3 & 1.60 & 4 & 1.97 \\
\hline E-32 & 4 & 1.93 & 2 & 1.06 \\
\hline 9738 & 4 & 2.01 & 4 & 1.97 \\
\hline
\end{tabular}

methods designed for homozygotic traits. They might result in different, even contrary conclusions. Thus, an innovative method has to be designed specifically for this trait.

Here, we designed an innovative method, i.e., a halfdiallel trial for equalizing all combinable chances and for minimizing the errors from different genetic background among the male and female parental lines. Under this half-diallel condition, the relationship is identified between the AFLP among the parental lines at different alleles and the heterotic performance of their corresponding $\mathrm{F}_{1}$ hybrids. Further, the favorable and unfavorable alleles related to heterosis were identified. In fact, those identified alleles were closely related to the combining ability between the male and female lines. Due to the various parental lines used in this method, those results could be representative for different hybrid combinations. This method should be practically useful for DNA marker assisted selection in hybrid rice breeding. Further, different genetic effects of the identified alleles could be evaluated based on genetic analysis though they could not be clearly mapped on the chromosomes.

In this study three kinds of genetic effects were evaluated (Fig. 3). Theoretically, both additive and dominant effects could appear in homozygosity, which should not belong to the heterosis category. As to the gene linkage, it is hard to have a homozygote in different alleles. For this reason, the dominant effect may not be easily gained through the homozygote as compared with the complementary dominant effect through heterozygotes. Thus, this genetic effect could also be important in heterotic exploitation in practice, which may belong to the heterosis category in terms of hybrid breeding. Of course, there are no arguments that the over-dominance is truly related to heterosis formation. In addition to the hybrid breeding program, due to the elite parents pyramided by a number of favorable additive and dominant alleles, we strongly recommend that they are selected as donors for improving parental lines.

Based on the 11 favorable and 8 unfavorable alleles identified from 18 parental lines, other parental genotypes have been evaluated in the number of the alleles (Table 3 ). The results were well identical to those obtained in hybrid rice programs, which should be a good reference for making hybrids and for choosing donors. Some of the parental lines containing relatively more favorable alleles and less unfavorable alleles should be useful in the hybrid rice breeding programs because they have a much higher combining ability.

\section{Acknowledgments}

This study is supported by both Zhejiang Natural Foundation, Zjejiang, P.R. China and IRRI/ADB Project "Development and Use of Hybrid Rice in Asia". The authors' thanks should be addressed to Prof. Q.Y. Tang for his computing assistance.

\section{Literature Cited}

Cerna,F.J., S.R.Cianzio, A.Rafalski, S.Tingey and D.Dryer (1997) Relationship between seed yield heterosis and molecular marker heterozygosity in soybean. Theor. Appl. Genet. 95: 460-467.

Diers, B.W., P.B.Mcketty and T.C.Ostorn (1996) Relationship between heterosis and genetic distance based on restriction fragment length polymorphism markers in oilseed rape (Brassica napus L.). Crop Sci. 36: 79-83.

Dudley,J.W. (1984) A method for identifying population containing favorable alleles not present in elite germplasm. Crop Sci. 24: 1053-1054.

Dudley,J.W., M.A.Saghai Maroof and K.Rufener (1992) Molecular 
marker information and selection of parents in corn breeding programs. Crop Sci. 32: 301-304.

Liu,X.C. and J.L.Wu (1998) SSR Heterogeneic patterns of parents for marking and predicting heterosis in rice breeding. Mol. Breed. 4: 263-268.

Martin,J.M., L.E.Talbert, S.P.Lanning and N.K.Blake (1995) Hybrid performance in wheat as related to parental diversity. Crop Sci. 35: 104-108.

Melchinger, A.E., M.Lee, K.R.Lamkey, A.R.Hallauer and W.L. Woodman (1990) Genetic diversity for restriction fragment length polymorphisms and heterosis for two diallel sets of maize inbreds. Theor. Appl. Genet. 80: 488-496.

Nagato, Y., A.Yoshimura and Conveners (1998) Report of the committee on gene symbolization, nomenclature and linkage groups. Rice Genet. Newsl. 15: 13-73.

Peng, J.Y., J.C.Glaszmann and S.S. Virmani (1988) Heterosis and isozyme diversions in indica rice. Crop Sci. 28: 561-563.

Peng,J.Y., S.S.Virmani and A.W.Julfiquar (1991) Relationship between heterosis and genetic divergence in rice. Oryza 28: 129133.

Saghai Maroof,M.A., K.M.Soliman, R.A.Jorgensen and R.W.Allard (1984) Ribosomal DNA spacer length polymorphism in barley: Mendelian inheritance, chromosomal location and population dynamics. Proc. Natl. Acad. Sci. USA 81: 8014-8018.

Stuber,C.W., S.E.Lincoln, D.W.Wolff, T.Helentjaris and E.S.Lander (1992) Identification of genetic factors contributing to heterosis in a hybrid from elite maize inbred lines using molecular mark- ers. Genetics 132: 823-839.

Virmani, S.S. (1994) Prospects of hybrid rice in the tropics and subtropics. In "Hybrid Rice Technology: New Developemnts and Future Prospects" S.S.Virmani (ed.), International Rice Research Institute, Manila Philippines. p. 7-19.

Wu,K.S. and S.D.Tanksley (1993) Abundance, polymorphism and genetic mapping of microsatellites in rice. Mol. Gen. Genet. 241: 225-235.

Xiao,J.H., J.M.Li, L.P.Yuan and S.D.Tanksley (1995) Dominance is the major genetic basis of heterosis in rice as revealed by QTL analysis using molecular markers. Genetics 140: 745-754.

Xiao,J.H., J.M.Li, L.P.Yuan and S.D.Tanksley (1996) Identification of QTL affecting traits of agronomic importance in a recombination inbred population derived from a subspecific rice cross. Theor. Appl. Genet. 92: 230-244.

Yu,S.B., J.X.Li, C.G.Xu, Y.F.Tan, Y.J.Gao, X.H.LI, Q.Zhang and M.A.Saghai Maroof (1997) Importance of epistasis as the genetic basis of heterosis in an elite rice hybrids. Proc. Natl. Acad. Sci. USA 94: 9226-9231.

Zanoni,U. and J.W.Dudley (1989) Use of $\mathrm{F}_{2}$ generation to identify inbred with favorable alleles not present in an elite hybrid. Crop Sci. 29: 583-589.

Zhang,Q.F., Z.Q.Zhou, G.P.Yang, C.G.Xu, K.D.Liu and M.A.Saghai Maroof (1996) Molecular marker heterozygosity and hybrid performance in indica and japonica rice. Theor. Appl. Genet. 93: $1218-1224$ 